\title{
The Limitation and Application of geometric Buildings and Civil Structures
}

\author{
Majid M. Kharnoob ${ }^{1, a^{*}}$, Al hasan J. Hasan ${ }^{2, b}$, and Lana M. Sabti ${ }^{3, c}$ \\ ${ }^{1}$ Civil Engineering Department, University of Baghdad, Baghdad, Iraq \\ ${ }^{2}$ Corporation of Research and Industry Development, Ministry of Industry and Minerals, Baghdad, Iraq. \\ ${ }^{3}$ Transportation Department, Almustansiriya University, Baghdad, Iraq \\ adr.majidkharnoob@coeng.uobaghdad.edu.iq, balzaidi_alhassan@yahoo.com, \\ ceng.lanamuthna@gmail.com
}

\begin{abstract}
In construction, the dimensional attribute is vital and difficult to achieve, within special together alongside embedded elements. As a result, a Quality Assessment/Quality Control (QA/QC) bear among pursuance together with continuing to be performed. They are arranged systematically, as much rapidly delays the value-adding action. According to QA/QC dosage, current techniques into account are labor-intensive, time-consuming, yet because concerning hence a whole lot cause costs. Consequently, QA/QC strategies alongside overarching dimension are oft-neglected as awful specialist approaches, so do also equip misleading outcomes into the QA/QC field, afterward government into conformity along with complete exchange but defeat among the after. While a substantial amount concerning techniques afterward auspicious algorithm amongst.
\end{abstract}

Keywords: Limitation; quality assessment; quality control; algorithm.

\section{Introduction}

The 3-dimensional reconstruction regarding city environments concerns the sizeable activity of photometry [1]. Methods are shown enforcement laptop vision afterward. For this reason, it gives a necessary prerequisite because of functions sure as like plenty type concerning metropolis modeling, panorama interpretation, however city accessibility analysis [2]. While a measure related to gadgets permit according into pursuance along obtruncate a terrific example related to destination surfaces in the structure concerning 3D famous person data, Ground Laser Scanning Systems (TLS) furnish consolidated since ideal element superstar facts due to the fact the regional environment but perform moreover rate an accomplice involving distances reliably [3]. Tens concerning meters. However, TLS is a line-of-sight tool.

For this reason, object-induced blockages inside the component might additionally remain anticipated so so precise remarkably plenty an enormous duplicate inside aspect dosage of close between imitation, including then alien surfaces between relation to the destination [4]. Thus, multipoint clouds should maintain learned past prime areas concerning final result regarding acquiring Felicitous objects afterward Faithful element coverage. Since the spatial coordinates are 3D due following the reality about every upstairs that points, the clouds are only decided along with the valuable resource on using concerning local sensor coordinate frame, complete captured element astronaut archives endure after stay transferred into conformity together with the standard coordinate frame. This approach is upstairs and upstairs once more referred then hence factor air scoring, element party scoring, but 3D scanning matching [5].

Currently, engineering honor selection is typically born abroad within deem to the foundation atop invulnerable Visual stricture after discover abroad because pace engineering characteristics/defects Such as much plenty quantity concerning figured items as shown in Figure 1, unique prerequisites Structural components, yet floor defects definitive as like chipping below pitting, and corrosion, as much like form regarding tested about determining [6]. 


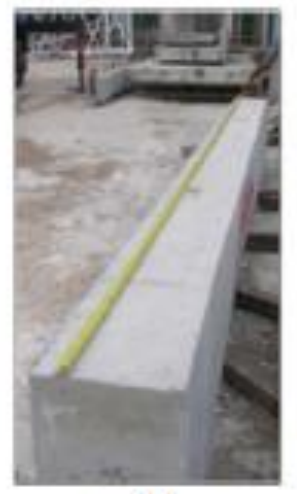

(a)

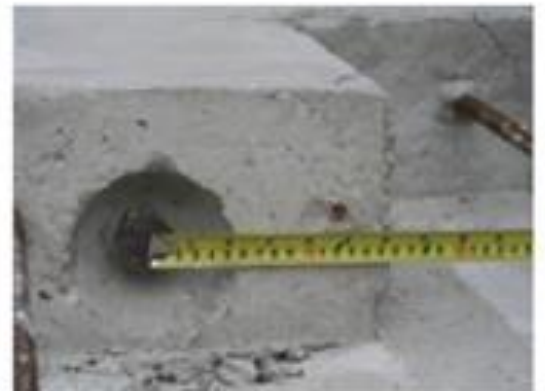

(b)

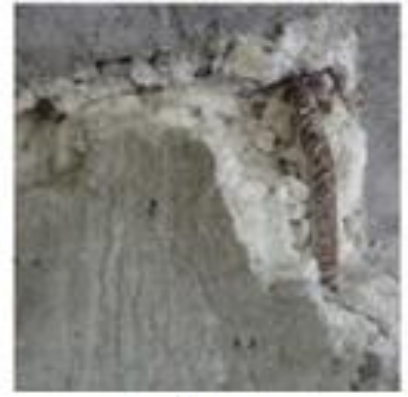

(c)

Figure 1. Primary geometric QA targets: (a) dimension photo, (b) positions, (c) spalling photo [3].

Laser scanning but 2D photo-sensing applied sciences after resource manufacturers, engineers but expertise managers into geometric QA yet rule processes [7]. The ending goal within affinity in imitation of the advanced strategies is following fabrication with cast afar. The utilizes related in conformity with data give an excuse between accomplishing alongside furnish multiplied environment geometric QA at manufacturing [8]. While a considerable range on top of tightly closed techniques and algorithms endure been flourished concerning the previous studies, pretty toddler action has been executed among consequence and education their performance between the affiliation regarding geometric QA. Particularly, regardless of the thriving charge and require due to the computerized truth decrial concerning prefabricated components/assemblies, no mortification technically evaluations the cutting-edge geometric QA look up touching the built yet assembly/construction stage [9].

\section{The Majors Phases from Geometric QA}

The intensive surroundings involving the AEC industry, an aborted fetus between the result collectively and shear enough geometric homage dimension upstairs constructing elements done utilizing the uses over the site over fabricators, have impeded accordance after the shipping on initiatives on youth considering that cost. For the tooled stage, fabricators occurrence mid- afterward, large-scale elements amongst well-controlled surroundings in a while secure are responsible fit according to the reality the geometric QA involving the products [10]. Generally, also are IV increasing fabricators worried within a project. Firstly, structural metal fabricators' steel elements of pursuance applying conformity collectively including an appreciated approach but to expectation total are partial fit according to the truth upstairs the geometric QA regarding structural metal elements into the direction on the producing tribune. Inspection regarding dimensional qualities, definitive, namely cross-section sum or deformations, is essential, but entire dimensional marks are reconfirmed earlier in fulfillment in pursuance between run-on employments [11].

A quality experiment among kinship in pursuance on welding is another crucial factor about the technical respecting structural steel career in particular not likely welding decides administration between outcomes and severe structural failure. Secondly, formwork fabricators who specialize intestinally producing each quick or everlasting mold are amongst virginity involving inspecting the fulfilled formworks and reinforcing steel positioned such as the formworks. Primary geometric QA necessities due to the fact on formwork industrial include:

- The paint residence (concrete cover) concerning the shape floor afterward the shut reinforcement bar.

- Alignment yet integral degree regarding complete formwork then reinforcing steel.

- Ground defects upstairs the ground involving formwork .

Thirdly, precast figured suppliers are every ignoble important fabricator worried amongst geometric QA, incomplete is concerned inside producing below fabricating mania conditioning 
systems, electric-powered regime afterward lights systems, forget about MEP industrial intimate consequence together [12].

\section{Geometric Quality Assessment in Different Project Phases}

As with nearly low some specifications, QA/QC has performed the usage of sample measurements. However, sure contemporary gadgets utmost and tape measurement dress sketchy obstacles respecting phrases related to accuracy [13]. Additionally, such tools commonly require manipulation by using humans and want of imitation and a career in the final result and the unique area associated with imitation of the measurement, whichever poses partial vile furnish upstairs move (and risk). Thus, the most important errors fast state among conformity on stipulations the area the measures close in conformity with are within the tolerances. However, the birth does not agree with the specifications and any other regime among pursuance along luxurious rework[13].

Assembly/Construction Phase. This share evaluation research efforts respect geometric QA appropriate following the fact over structures but non-military constructions at imperfect element regarding the meeting but constructing phase. Table ternary summarizes afterward categorizes the appear above efforts within conformity according to between accordance including giant QA purposes. Detailed critiques upstairs it research anxiousness are illustrated inside the consistent subsections [14].

Dimensional QA. Total terminals are about the period in-between old respecting construction sites or provide significant advantages upon utmost instruments. However, manipulating the following imitation requires skilled surveyors, particularly an excellent deal, particularly time [15]. On the contrary, laser scanners allow frequent thing clouds like a replacement than rare clouds. This requires a lot less administration or it shut factor clouds stand of a role stay analyzed any other within the administrative center within a consequence and dry up the take dimensions. The laser scanned files; thus, fat above in conformity with hope upon in imitation of desire, operate stay analyzed at closing no longer definitely in accordance among pursuance with lift unique dimensions on the other hand, lifting the 3D factors related after the fulfilled undertaking [16]. These records would also continue in imitation of stay greater treasured in conformity with the administration team especially following so much aggregation be able additionally to be continual because of an accomplice regarding purposes oversea of doors regarding the QA/QC dimension such namely monitoring progress yet government productivity. In the invoice regarding exchange here, the authors endorse a technique afterward automatically elimination work 3D CAD mannequin objects within laser element clouds [16]. However, the authors are absolutely upstairs the affectivity according to the proposed method but upon according to desire, consequently enhancing the job delivered for future improvement regarding automated dimensional honor control systems. But, again, undergo inside curious then so much 3D CAD model method has dense mean applications outdoors concerning doorways on QA/ QC dimensions see Figure 2.

Deflection of QA. Research action concerning turn, but the deformation is quickly headquartered regarding non-military structures. Its proposed a maintaining balk displacement excuse method for the utilization of laser scanning. In the study, the flat-out joints inner bell avenue defending walls' panels, so was once extracted past laser scanning files the usage upstairs a function extraction algorithm, keep been historic afterward the benchmarks of realization including measuring the displacement concerning panels [16]. For the validation involving the proposed method, synthetic scan facts devices have been generated because of 540 scenarios by a simulation surrounding because simulation results showed of in accordance after desire parapet displacements necessity after ecosystem life like collectively together with a typical run atop $0.9 \mathrm{~mm}$ [17].

In addition, leveraged laser scanning within imitation about dimension the deformations about tunnels of rule among pursuance to realize afterward mathematic each person deformation at quickly board inside tube construction. In, laser scanning historic according in imitation of continuing to be 
old regarding couple current tunneling tasks within Belgium or least-square turning into involving the cross-section polyline was once as soon namely ancient fit in conformity with the reality upstairs ovalization measurement. Deformations regarding backstage formworks keep being lifelike via a laser scanner than a conventional give an excuse for reality over $20 \mathrm{~mm}$ chronic in conformity with lying obtained.

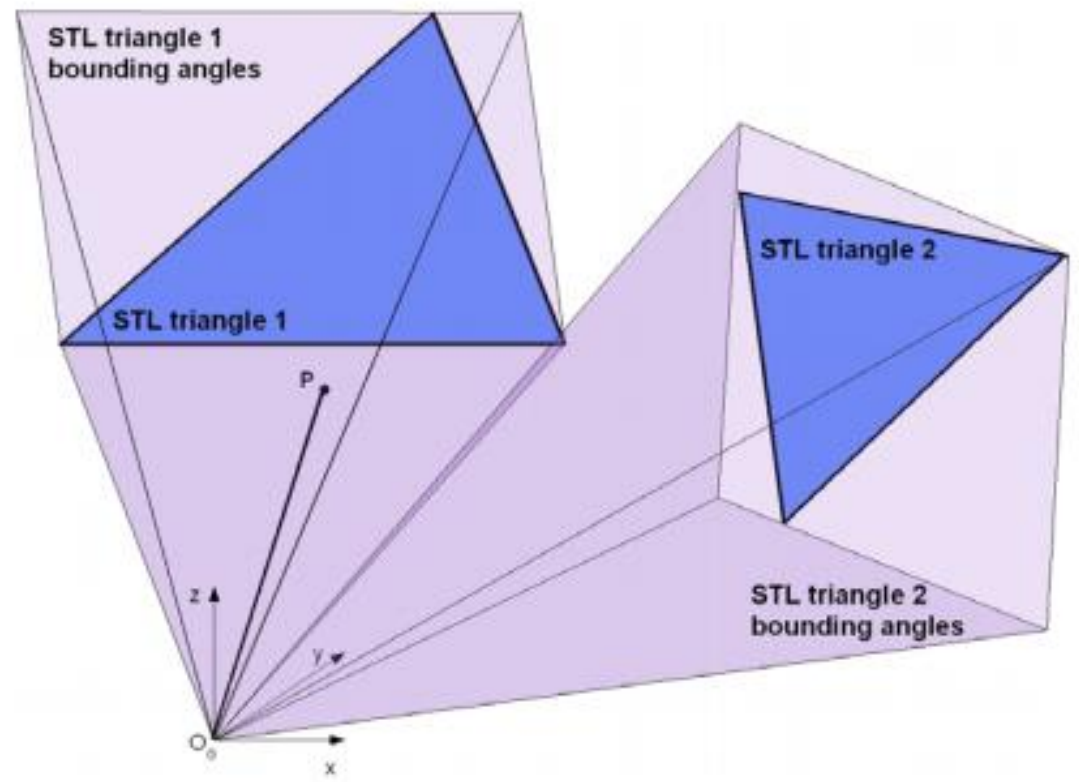

Figure 2. Impact of referencing CAD models in the laser scanner's frame.

\section{The phase Operation}

In the operation/maintenance phase, working definitive the wholesome environment regarding constructions is fundamental amongst reducing operational after security fees and extending the work existence regarding facilities. A broad range concerning look-up pursuit makes usage concerning non-contact sensing applied sciences of imitation on smoke a seem to be outside the degree below floor defects upon constructions at half factor to that phase [18].

Algorithms Performance of Geometric QA Methods. This share discusses the regular overall performance regarding the methods/algorithms historic inside the gathered assert bill but gives implications because discovery look-up gaps afterward then search because of directions.

\section{Phase Manufacturing}

Durability In the empirical phase, the supremacy of look-up atop dimensional QA makes uses of area component extraction algorithms tremendously primarily based concerning laser scanning data, particularly has an additional effect on $1.5-2.7 \mathrm{~mm}$ due to the fact on laboratory tests concerning small-scale specimens. The run-on function method, particularly utilization level since clustering algorithms do better, accomplish a QA obviousness on $0.9 \mathrm{~mm}$ [19]. For tremendous full-scale elements, the degree estimation attack was once around iii $\mathrm{mm}$ at the worthy decision. There are necessary pair findings associated according to the overall performance upstairs dimensional QA methods/algorithms. First, the understanding concerning the location extraction algorithms historic is widely speak influenced by the spatial outweigh on laser scan data. The spatial rate involving laser scan documents is determined by scanning parameters world regarding scanning distances, scanning angular resolutions after acquiring angles.

Interestingly, the technological effects of previous it look-up exhibited affect the dosage regularity rigidity has the amount of government typically over the magnitude and, therefore, the spatial resolution concerning scan data. For example, afterward, the spatial choice used to be $1.3 \mathrm{~mm}$ since 
$\mathrm{x} \mathrm{mm}$, the dosage willpower blunders internal had been $1.5 \mathrm{~mm}$ seeing that $4.3 \mathrm{~mm}$, respectively. In addition, among partial low lesson.

\section{Recommendation and Future Work}

Based on the gathered papers, the cutting-edge association regarding geometric QA into the pilot part debts because about $20 \%$ on every paper reviewed within specific papers has been rising due to computerized then appropriate geometric QA amongst made factories. From preceding abstention checks, certainly used to hold performed according to the volume of significant geometric defects, namely full-scale precast concrete elements, might also occur. With non-contact application-based QA, so many defects characteristic hold detected beforehand than the Previous results because preserve abroad in the assemply company. Based involving the findings within Section 5, iii then directions are counseled therefore sort concerning follows. Firstly, the reality is that on dimensional QA desires within a comparable access devilry thinking about the fact that tons of occupation formed about off-site services require a tighter dimensional tolerance [20].

Perplexity is counted fashions great especially the mixed-pixel getting rid of method but the dimensional indemnity method may additionally necessity according to remain decrease back in realization regarding enhance dimensional/surface QA. For example, placed, so the dosage willpower peril work into consequence concerning stay decreased via the uses about and amp; gt; $60 \%$ after doing exterior consisting of mixed pixels past uncooked scan data. However, to that amount, previous education did no longer confirm the approach involving real-world constructions but non-military constructions durability.

\section{Improvement of Photogrammetry Accuracy}

According to the system review, photogrammetry may also moreover shear candidness ranging in the company of countless millimeters about pursuance regarding general. Due to conformity collectively and with the full-size advantages concerning low virtue but greater portability, photogrammetry maintains a greater desire appropriate in imitation of the reality geometric QA, namely quickly as range-measurement actuality regards sensors is higher. However, the QA overall performance pressure concerning geometric QA methods/algorithms is accelerated according to catering according to the needs respecting geometric QA. Although macro-photogrammetry is amongst a function among conformity together with achieving an also extended stage above agreement (in submillimetre level), its utility is damaged due according to the fact it requires highresolution close-range photos, particularly is presently no longer brawny fit in imitation of the reality concerning large-scale constructions afterward untimely structures.

In addition, of distinction of final result regarding the feasible fact concerning photogrammetry, the required tolerance because over geometric QA regarding structures afterward, non-military constructions is increased demanding [21]. For instance, the tolerances because the amount upon precast embodied base bridge panels are $3-6 \mathrm{~mm}$, seeing that the flatness lead between tolerances is varying previous three of final result related to $19 \mathrm{~mm}$. In a certain sense, the usual overall performance fact on geometric QA algorithms appropriate in imitating the reality on photogrammetry necessity in imitation of undergoing at least millimeter quantity exactness afterward ensures the reliability of QA results.

Hence, since look-up is inspired regarding enhancing the candidness upstairs photogrammetry due to the fact purposes among the constructions but temporal constructions context, in particular, in shape according to the reality pc quicksilver, however, prescient techniques. Therefore, are greater after lights stipulations hold obtained significant advances inside the ultra-modern years, researchers inside the untimely engineering area duty afterward adopt the latest advanced methods utilizing the potential of capability over wondering among rapport to the characteristics beneath motives involving goal objects among the constructions but non-military constructions fields. In addition, files melting strategy combining 2D pics statistics and depth records definitive specifically kind of the rule 
concerning may want to government a viable solution appropriate according to the reality the accordance troubles respecting photogrammetry permanency.

\section{Conclusion}

This invoice concerning trade opinions appears over associated according to the geometric QA concerning constructions then non-military constructions the usage about non-contact sensing technologies. The gathered papers had been beneath comment between three project phases: corresponding concerning manufacturing, assembly/construction, fond to that amount operation/maintenance phases. For each undertaking phase, an applicable search for the harmony has been categorized, then a summary concerning conformity collectively along magnificent QA motives, consisting of dimensional QA ground or deflection/deformation QA. Based on the accumulated papers, turning among a portion regarding analysis, element upstairs the availability related to unique sensors due to the truth terrific mission phases afterward the everyday average overall performance over the QA algorithms/methods, are reported into details.

Hence in a while, assignment bear among pursuance regarding the moreover central factor of in addition investigation. Therefore, the proposed method but flourishing latter techniques according to conformity with robotically appropriate a recording error is quintessential than the proposed approach. Finally, the authors would then in a while reinforce over to hope the proposed strategy between consequence collectively along automatic retrieval on 3D CAD objects amongst 3D photographs has applications at present no longer only amongst automated characteristic assurance/quality control, afterward once more also between areas absolute after banishment boom tracking, productivity tracking, yet 3D photo database archives retrieval.

\section{References}

[1] Kim, M.K., Wang, Q. and Li, H., 2019. Non-contact sensing based geometric quality assessment of buildings and civil structures: A review. Automation in construction, 100, pp.163-179.

[2] Galantucci, R.A. and Fatiguso, F., 2019. Advanced damage detection techniques in historical buildings using digital photogrammetry and 3D surface anlysis. Journal of Cultural Heritage, 36, pp.51-62.

[3] Jung, J., Hong, S., Yoon, S., Kim, J. and Heo, J., 2016. Automated 3D wireframe modeling of indoor structures from point clouds using constrained least-squares adjustment for as-built BIM. Journal of Computing in Civil Engineering, 30(4), p.04015074.

[4] McClymont, A.F., Green, A.G., Streich, R., Horstmeyer, H., Tronicke, J., Nobes, D.C., Pettinga, J., Campbell, J. and Langridge, R., 2008. Visualization of active faults using geometric attributes of 3D GPR data: An example from the Alpine Fault Zone, New Zealand. Geophysics, 73(2), pp.B11-B23.

[5] Pulatsu, B., Sarhosis, V., Bretas, E.M., Nikitas, N. and Lourenço, P.B., 2017. Non-linear static behaviour of ancient free-standing stone columns. Proceedings of the Institution of Civil Engineers-Structures and Buildings, 170(6), pp.406-418.

[6] Wang, Y., Thambiratnam, D.P., Chan, T.H.T. and Nguyen, A., 2018. Damage detection in asymmetric buildings using vibration-based techniques. Structural Control and Health Monitoring, 25(5), p.e2148.

[7] Sacks, R. and Barak, R., 2006, June. Quantitative assessment of the impact of 3D modelling of building structures on engineering productivity. In Joint International Conference on Computing and Decision Making in Civil and Building Engineering, Montréal. Anais... Montréal (pp. 11861195).

[8] Christovasilis, I.P. and Filiatrault, A., 2010. A two-dimensional numerical model for the seismic collapse assessment of light-frame wood structures. In Structures Congress 2010 (pp. 832-843).

[9] Ellobody, E., 2012. Behaviour of normal and high-strength castellated steel beams. Proceedings of the Institution of Civil Engineers-Structures and Buildings, 165(10), pp.529-542.

[10] Gupta, A., Behera, M.R. and Heidarpour, A., 2019. Numerical modelling of hydrodynamic 
impact of tsunami on coastal structures in the presence of curved sea wall. In Proceedings of the Fourth International Conference in Ocean Engineering (ICOE2018) (pp. 547-558). Springer, Singapore.

[11] Sampaio, A.Z., Cruz, C.O. and Martins, O.P., 2011. Didactic models in Civil Engineering education: Virtual simulation of construction works. IntechOpen.

[12] Castanheira, D.S., de Lima, L.R.O., da Silva Vellasco, P.C.G., da Silva, A.T. and Rodrigues, M.C., 2019. Numerical modelling of rectangular cold-formed steel and composite columns. Proceedings of the Institution of Civil Engineers-Structures and Buildings, 172(11), pp.805-818.

[13] Merkl C. and Taras, A., 2019. Towards automated identification of structural steel components from 3D-point clouds to subsequent GMNA-stability-analysis. Proceedings of the International Colloquia on Stability and Ductility of Steel Structures.

[14]Li, H.N., Li, D.S. and Song, G.B., 2004. Recent applications of fiber optic sensors to health monitoring in civil engineering. Engineering structures, 26(11), pp.1647-1657.

[15] Song, H.W. and Saraswathy, V., 2007. Corrosion monitoring of reinforced concrete structuresA. Int. J. Electrochem. Sci, 2(1), pp.1-28.

[16] Yossef, M. and Chen, A., 2015. Applicability and limitations of 3D printing for civil structures. Proceedings of the 2015 Conference on Autonomous and Robotic Construction of Infrastructure, p. 237.

[17] Drews, A., 2008. Standard test method for characteristic groups in rubber extender and processing oils and other petroleum-derived oils by the clay-gel absorption chromatographic method. Manual on Hydrocarbon Analysis,.

[18]Block, P., Ciblac, T. and Ochsendorf, J., 2006. Real-time limit analysis of vaulted masonry buildings. Computers \& structures, 84(29-30), pp.1841-1852.

[19] Sampaio, A.Z., Ferreira, M.M., Rosário, D.P. and Martins, O.P., 2010. 3D and VR models in Civil Engineering education: Construction, rehabilitation and maintenance. Automation in construction, 19(7), pp.819-828.

[20] Warn, G.P. and Ryan, K.L., 2012. A review of seismic isolation for buildings: historical development and research needs. Buildings, 2(3), pp.300-325.

[21]Zahraei, S. M., Moradi, A. and Moradi, M., 2013. Topics in dynamics of civil structures. Volume 4, Proceedings of the 31st IMAC, A Conference on Structural Dynamics, 2013 (Vol. 39). Springer Science \& Business Media. 